\title{
Physicians Experience with and Expectations of the Safety and Tolerability of WHO-Step III Opioids for Chronic (Low) Back Pain: Post Hoc Analysis of Data from a German Cross-Sectional Physician Survey
}

\author{
Michael A. Ueberall, ${ }^{1}$ Alice Eberhardt, ${ }^{2}$ and Gerhard H. H. Mueller-Schwefe ${ }^{3}$ \\ ${ }^{1}$ Institute for Neurological Sciences, Nordostpark 51, 90411 Nuernberg, Germany \\ ${ }^{2}$ Mundipharma GmbH, Mundipharmastraße 2, 65549 Limburg, Germany \\ ${ }^{3}$ Interdisciplinary Center for Pain and Palliative Care Medicine, Schillerplatz 8/1, 73033 Goeppingen, Germany \\ Correspondence should be addressed to Michael A. Ueberall; michael.ueberall@ifnap.de
}

Received 29 July 2015; Accepted 21 September 2015

Academic Editor: Steve McGaraughty

Copyright (C) 2015 Michael A. Ueberall et al. This is an open access article distributed under the Creative Commons Attribution License, which permits unrestricted use, distribution, and reproduction in any medium, provided the original work is properly cited.

Objective. To describe physicians' daily life experience with WHO-step III opioids in the treatment of chronic (low) back pain (CLBP). Methods. Post hoc analysis of data from a cross-sectional online survey with 4.283 Germany physicians. Results. With a reported median use in $17 \%$ of affected patients, WHO-step III opioids play a minor role in treatment of CLBP in daily practice associated with a broad spectrum of positive and negative effects. If prescribed, potent opioids were reported to show clinically relevant effects (such as $\geq 50 \%$ pain relief) in approximately 3 of 4 patients (median $72 \%$ ). Analgesic effects reported are frequently related with adverse events (AEs). Only $20 \%$ of patients were reported to remain free of any AE. Most frequently reported AE was constipation (50\%), also graded highest for AE-related daily life restrictions (median 46\%). Specific AE countermeasures were reported to be necessary in approximately half of patients (median 45\%); nevertheless AE-related premature discontinuation rates reported were high (median 22\%). Fentanyl/morphine were the most/least prevalently prescribed potent opioids mentioned (median 20 versus 8\%). Conclusion. Overall, use of WHO-step III opioids for CLBP is low. AEs, especially constipation, are commonly reported and interfere significantly with analgesic effects in daily practice. Nevertheless, beneficial effects outweigh related AEs in most patients with CLBP.

\section{Background and Introduction}

WHO-step III opioids are increasingly prescribed for the treatment of chronic nonmalignant pain (CNMP) in industrialized countries [1-3]. Based on German health insurance data, overall consumption of WHO-step III opioids by patients suffering from CNMP increased from 24.1 million defined daily doses (DDD) in 2000 to 107.6 million in 2010 [4]. Underlying reason for this 4 -fold increment is increases in treatment prevalence (from 0.18 to $0.86 \%$ of the German population, mainly due to an increased use in CNMP such as back pain), and treatment duration (e.g., the proportion of patients receiving opioids for longer than 90 days increased from $4.3 \%$ in 2000 to $7.5 \%$ in 2010) [4].

The increasing popularity of WHO-step III opioids for CNMP is frequently explained by their pharmacological interaction with endogenous pain relieving systems (going hand in hand with efficacy in a broad spectrum of painful conditions), the lack of analgesic ceiling effects (i.e., doses can be escalated until either desired or limiting effects are reached), and the absence of a significant toxicity to internal organs; although side effects from these drugs are common, they are usually transient and reversible upon treatment discontinuation in contrast to those observed with quite 
a few nonopioid analgesics (e.g., acetaminophen, dipyrone) $[5,6]$ and most of the nonsteroidal anti-inflammatory drugs (NSAIDs) [7-11].

When used properly, WHO-step III opioids are known to counteract pain that follows surgery, to palliate suffering associated with advanced cancer and to mitigate CNMP. However, while their use for the treatment of postsurgical and cancer-related pain is generally accepted and the available scientific evidence is graded as sufficient to justify their use, the framework for a general recommendation to use them in patients suffering from CNMP is insufficient. Due to the limited evidence from controlled clinical trials, the lack of long-term effectiveness, tolerability, and safety data for treatments lasting longer than three months, and the potential interaction of opioids with endogenous factors leading to individually more or less critical biological, psychological, and social implications, current treatment guidelines uniformly recommend a conservative use of WHO-step III opioids for CNMP [12-16].

Long-term daily use of opioids is associated with significant side effects (e.g., constipation and opioid-induced bowel dysfunction, dizziness, concentration problems, urinary retention, and itching) in the majority of patients, which, even if not associated with a relevant organ toxicity, significantly interfere with patient outcome and pain relieving effects. In addition, a low but nevertheless significant risk exists to develop an opioid use disorder (OUD) characterised by clinical signs/symptoms of either drug abuse, dependence, and/or addiction, even in those patients for whom the treatment indication is based on generally agreed considerations and who take their opioids as directed. Available data suggests that the risk for an OUD varies with patient history, psychosocial factors, and comorbid psychopathology and increases with longer treatment duration, use of immediate release preparations, higher opioid dose, and polymedication [17]. However, even the absence of all these risk-factors does not necessarily eliminate any risk for the development of an OUD.

Moreover, few opioid patients develop an opioid tolerance (probably due to a desensitization and/or downregulation of opioid receptors), in which the ongoing exposure to the drug results in a decline of the primarily achieved effects over time [18]. And beyond that phenomenon (which is usually counteracted either by an increment in opioid dose or by an opioid rotation), opioids might also induce neuroplastic changes in the peripheral and central nervous system (CNS) that lead via a sensitization of pronociceptive pathways to an opioid-induced hyperalgesia [19]. Unfortunately, this complication presents clinically at first sight similarly to the tolerance phenomenon and is consecutively followed by reactive dose escalating countermeasures, which vice versa open the door to a vicious circle of treatment changes that do not only decrease the chance for a beneficial outcome but also increase the risk for an OUD. In combination with the multifactorial reasons underlying CNMP, this complexity of opioid-effects may explain why WHO-step III agents can be remarkably helpful in many patients with CNMP, while in others they either do not work or even increase the problems over time.
In response to the increasing prescription rates of strong acting opioids for patients with CNMP, experts and guidelines critically state that the long-term attested underuse of opioids at the end of the 20th century has now been transformed into a wrongful over- and a critical misuse and that the physicians responsible for that either do not know about the multifractal peculiarities of opioids and related risks in CNMP or even ignore any evidence-based treatment recommendations by overestimating positive and underestimating negative opioid-related effects interfering with patient outcome.

To highlight the silent reproach underlying these statements and to gain further insight into the real-life experience and expectations of physicians treating CNMP patients with WHO-step III opioids, the steering committee of the German Pain League, Germany's largest pain patients self-regulating community, asked the German Pain Association to perform a post hoc analysis of so far unpublished data of a large cross-sectional survey of 4.283 German physicians originally performed in 2012 and to focus now on data addressing global tolerability and safety aspects of treatments with WHO-step III opioids in CNMP.

\section{Methods}

Between August and September 2012, the German Pain Association initiated an online census and invited nearly 12,000 German physicians, known to have a special focus in pain patients and pain therapy, to participate in a cross-sectional national survey entitled CROSSECCO (cross-sectional noninterventional evaluation of physicians' preferences and experiences with strong acting opioid analgesics for the treatment of chronic nonmalignant pain) and to report about their experience with and their expectations of WHO-step III opioids in CNMP. Methodology and results (focusing on general and national aspects) of this survey have been published in 2014 [20]. However, due to the increasing concerns about safety issues of WHO-step III opioids in patients suffering from CNMP [especially chronic (low) back pain, CLBP] and in reaction to the proposal of the German Pain League the German Pain Association commissioned a post hoc analysis of so far unpublished data of this survey focusing now on global tolerability and safety aspects of WHO-step III opioids for the treatment of CLBP that outreach national conditions. The concept for this post hoc analysis has been developed by MAU and GHHMS and has been finally approved by the steering committees of the German Pain Association and the German Pain League.

The original online survey consisted of 157 questions addressing the individual profile of participating physicians (e.g., age, years of experience, specialisation, additional qualifications in pain therapy, and type of practice setting), their patients (e.g., number of patients with pain by condition, duration of pain and stage of chronification, and biological and psychological pain characteristics), and their experience with as well as expectations of various opioids for different pain conditions. Beside general information, a considerable proportion of questions focused on the use of WHOstep III opioids for the treatment of CLBP and addressed 
patient baseline characteristics (e.g., history, pain duration, intensity, localization, chronification, functional handicaps, restrictions in daily life and quality of life, and comorbidities), pretreatment data, type and duration of opioid treatment (dose, usage characteristics, etc.), opioid-related treatment effects (e.g., pain relief, improvements with respect to quality of life and daily life activities), and safety and tolerability aspects.

Dependent on type of question, participants were asked either to select one of several predefined verbal or numerical options to answer a (multiple-choice type) question or to move a slider on a $100 \mathrm{~mm}$ visual analogue scale (VAS, ranging from $0=$ none to $100=$ all) to quantify the percentage of patients to whom a distinct question or statement belonged.

Statistics were done descriptively only and with respect to the dimensions of the scales used (i.e., for categorical scales absolute and relative (if necessary adjusted) percentages were evaluated and for ordinal scales mean, standard deviation, median, and percentiles). For graphical analyses box-andwhisker diagrams were used (with the bottom and top of the box defined by the first and third quartiles, the band inside by the median, and the whiskers representing the 2.5 th and 97.5th percentiles). All analyses were done with the "as reported data set" without any data imputation for missing parameters. Average ( \pm standard deviation) participation rate of physicians per question was $98.9( \pm 0.8)$ percent (median $99.1 \%)$. Statistical procedures were applied if appropriate to evaluate the significance of differences found not to confirm any predefined hypotheses. Test procedures used were the Chi-Square test for categorical and the Student $t$-test for ordinal scaled variables.

\section{Results}

3.1. Relevance of WHO-Step III Opioids for CLBP. As reported by the participants of this survey, WHO-step III opioids have a definitive but subordinate meaning among the spectrum of pharmacological and nonpharmacological treatments usually used for patients suffering from CLBP (see Figure 1).

With respect to the spectrum of those 17 treatment approaches for CLBP evaluated in the original survey, reported prescription rates of WHO-step III opioids were low and rank on average (mean \pm SD) with $22.7 \pm 17.2 \%$ (median $17 \%$ ) on position 9 of the list. Reported prescription rates of NSAIDs/Cox-2s for CLBP were with $55.1 \pm 23.8 \%$ (median $60 \%)$ significantly higher $(p<0.001$; rank 1$)$, as well as those for nonopioid analgesics (35.6 \pm 20.9 , median 30\%; $p<0.001$; rank 3), WHO-step II opioids (28.3 \pm 16.0 , median $27 \%$; $p=0.001$; rank 4), coanalgesics (29.8 \pm 22.3 , median $24 \%$; $p<0.001$; rank 5), muscle relaxants $(25.8 \pm 18.5$, median $21 \% ; p<0.001$; rank 6 ), and selective nerve root blocks/facet joint injections $(27.8 \pm 23.0$, median $20 \%$; $p<0.001$, rank $7)$. Nonpharmacological approaches such as physiotherapy and physical treatments were with reported prescription rates of $47.7 \pm 25.9 \%$ (median $45 \% ; p<0.001$; rank 2) and $25.6 \pm 20.5 \%$ (median 20\%; $p=0.027$; rank 8) which are significantly more commonly used for CLBP patients than WHO-step III opioids, whereas chiropractic procedures

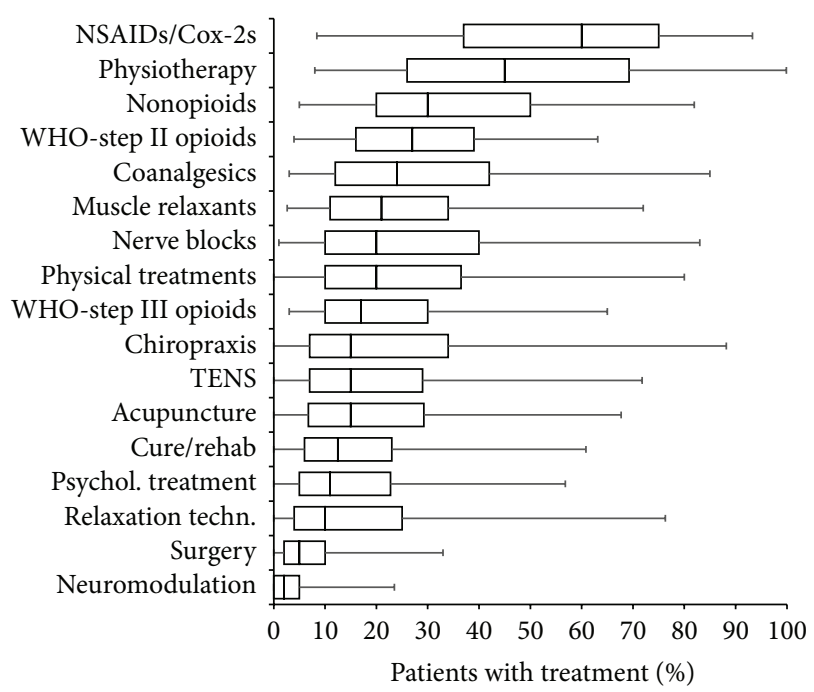

FIGURE 1: Frequency of use of different pharmacological and nonpharmacological treatment approaches for chronic low back pain sorted by percentage in descending order. Parameters shown are box-and-whisker diagrams (with the bottom and top of the box defined by the first and third quartiles, the band inside by the median, and the whiskers representing the 2.5th and 97.5th percentiles). NSAIDs: nonsteroidal anti-inflammatory agents; Cox2s: selective Cox-2 inhibitors; WHO: World Health Organization; TENS: transcutaneous electric nerve stimulation.

$(24.5 \pm 24.5 \%$, median $15 \%)$, transcutaneous electrical nerve stimulation ( $21.4 \pm 19.5 \%$, median $15 \%)$, and acupuncture $(20.8 \pm 19.3 \%$, median $15 \%)$ were reported to be comparably often prescribed ( $p=\mathrm{ns})$.

3.2. Opioid Treatment Characteristics. With reported prescription rates of $26.0 \pm 20.8 \%$ (median $20 \%$ ) fentanyl was the most frequently used WHO-step III opioid for CLBP (see Figure 2), followed by oxycodone/naloxone (19.9 \pm 18.5, median: $15 \%)$, oxycodone (17.8 \pm 17.1 , median $13 \%)$, hydromorphone $(13.9 \pm 13.6$, median $10 \%)$, buprenorphine $(13.8 \pm 15.0$, median $10 \%)$, morphine $(13.7 \pm 16.8$, median $8 \%)$, and tapentadol $(7.3 \pm 12.5$, median $2 \%)$, a WHO-step III centrally acting analgesic with a dual mode of action (opioid/NA reuptake inhibition).

With $16.5 \pm 18.5 \%$ (median $11 \%$ ), physicians reported monotherapy with opioids to be more the exception than the rule. In most patients, survey participants reported to give WHO-step III opioids in combination with other analgesics such as NSAIDs/Cox-2s (23.4 \pm 15.8 , median $21 \%)$, adjuvant agents $(21.9 \pm 13.7$, median $20 \%)$, or nonopioid analgesics $(18.5 \pm 17.6$, median $17 \%)$. Use of WHO-step III opioids as part of a multimodal treatment concept in combination with several other approaches has been reported for $23.6 \pm 17.4 \%$ (median 20\%) of CLBP patients.

Treatment duration varied with respect to treatment effects achieved and adverse effects experienced (see below). Average proportion of patients reported to receive WHO-step III opioids for less than 4 weeks was $9.4 \pm 10.2 \%$ (median $6 \%$ ), $16.9 \pm 10.8 \%$ (median $16 \%$ ) for $1-3$ months, $19.4 \pm 9.2 \%$ 


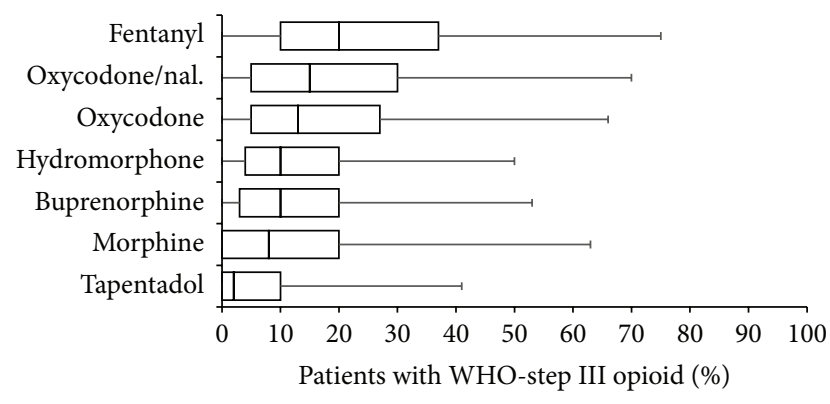

FIGURE 2: Frequency of use of different WHO-step III opioids for the treatment of chronic low back pain sorted by percentage in descending order. Parameters shown are box-and-whisker diagrams (with the bottom and top of the box defined by the first and third quartiles, the band inside by the median, and the whiskers representing the 2.5 th and 97.5 th percentiles).

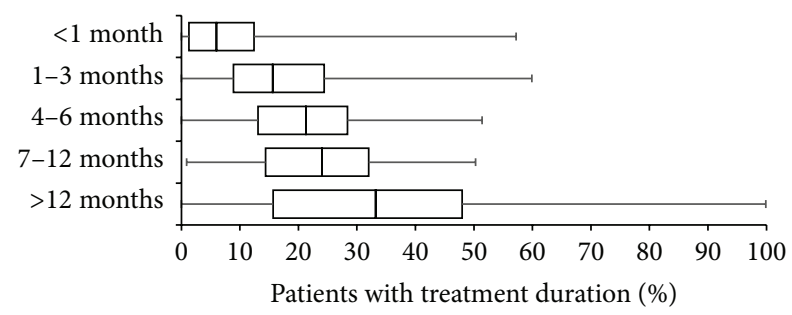

FIGURE 3: Treatment duration of patients with chronic low back pain with WHO-step III opioids. Parameters shown are box-and-whisker diagrams (with the bottom and top of the box defined by the first and third quartiles, the band inside by the median, and the whiskers representing the 2.5 th and 97.5 th percentiles).

(median 21\%) for 4-6 months, $21.4 \pm 9.8$ (median 24\%) for 7-12 months, and $32.9 \pm 19.4$ (median 33\%) for treatments longer than 12 months (see Figure 3).

3.3. Pain Relief and Related Treatment Effects. Overall, beneficial treatment effects achieved with the introduction of WHO-step III opioids were reported to be satisfying (see Figure 5). A favourable response to the treatment has been reported for $69.6 \pm 23.8 \%$ of patients (median $75 \%$ ) and for $67.5 \pm 23.7 \%$ (median $72 \%$ ) survey participants reported a pain relief of at least $50 \%$ versus pretreatment. Average percentages of patients for whom a clinically relevant improvement with respect to their daily life activities and their overall quality of life has been reported were $64.8 \pm$ $23.6 \%$ (median 68\%) and $64.6 \pm 23.0 \%$ (median $70 \%$ ), respectively. For $54.0 \pm 25.3 \%$ (median $55 \%$ ) of CLBP patients, physicians reported that the use of WHO-step III opioids and the pain relief achieved with their introduction paved the way to conduct or participate in alternative treatment approaches; patients were not able to do so before (e.g., physiotherapy, sport, and cure/rehab). For one-third of CLBP patients (38.8 \pm 26.8 , median $37 \%$ ), survey physicians reported that the beneficial effects achieved with WHO-step III opioids continued beyond the treatment period and persisted despite treatment discontinuation. Vice versa, for one-third of patients $(34.3 \pm 25.8$, median $29 \%)$ significant worsening of pain intensity and related issues has been reported after treatment discontinuation.

3.4. Safety and Tolerability Aspects. Adverse effects (AEs) with WHO-step III opioids were reported to be frequent (see Figure 6). On average, transient/short-term AEs ( $\leq 2$ weeks) were reported for $18.2 \pm 11.3$ (median $16 \%$ ) of patients, intermediate AEs (lasting 3-8 weeks) for $44.7 \pm 26.1$ (median $45 \%$ ), and persistent/long-term AEs (>8 weeks) for $17.6 \pm$ $9.8 \%$ (median 19\%). Only for one in five patients $(25.3 \pm 23.2$, median 20\%), physicians reported no relevant adverse events in response to the treatment with a WHO-step III opioid.

The most frequently stated AE was constipation with an average reporting rate of $49.1 \pm 24.9 \%$ (median $50 \%$ ), followed by somnolence (26.6 \pm 19.6 , median $21 \%$ ), dry mouth $(22.7 \pm 20.2$, median $18.5 \%)$, reduced performance $(17.8 \pm 17.2$, median $13 \%)$, and neurological (15.7 \pm 14.7 , median $11 \%)$ and mental problems (13.3 \pm 12.7 , median $10 \%$; see Figure 7). Overall, AE-related restrictions were graded highest for constipation $(46.1 \pm 23.5$, median $46 \%)$, followed by opioid-induced performance impairments $(15.9 \pm 17.1$, median 10\%), urinary retention (11.3 \pm 19.8 , median $4 \%)$, itching $(9.2 \pm 14.6$, median $4 \%)$, and endocrine disorders $(6.1 \pm 11.7$, median $1 \%)$.

The percentage of patients reported to need specific treatments to countermeasure opioid-related side effects was $58.4 \pm 27.4 \%$ (median $45 \%$ ). One-third of those patients $(35.6 \pm 17.8$, median $36 \%)$ were reported to put the treatment with WHO-step III opioids generally in question due to the side effects experienced, and one of five patients (20.2 \pm 14.8 , median 22\%) discontinued prematurely. Consecutively, AErelated problems due to WHO-step III opioids are frequently noted: for $6.2 \pm 9.8 \%$ (median $2 \%$ ), respectively, $7.3 \pm 11.5 \%$ (median 3\%) of patients, survey participants reported AErelated familial and social problems and emotional and physical distress for further $7.3 \pm 12.9 \%$ (median $2 \%$ ) and $8.8 \pm$ $12.5 \%$ (median $4 \%$ ) in addition; $12.3 \pm 15.5 \%$ (median $7 \%$ ) of CLBP patients treated with strong opioids were reported to be temporarily, $8.6 \pm 13.5 \%$ (median $3 \%$ ), persistently unable to work due to treatment-related AEs, and $3.7 \pm 8.2 \%$ (median 2\%) lost their job due to opioid-related side effects (see Figure 8).

3.5. Prevalence of Tolerance, Hyperalgesia, and Opioid Use Disorder. On average, survey participants reported for onefourth of patients $(26.2 \pm 19.9$, median $21 \%)$ the need for recurrent dose adjustments during opioid maintenance treatment to keep analgesic effects stable (see Figure 4). Nevertheless, for one in five patients $(20.2 \pm 17.9$, median $16 \%)$ a loss of efficacy over time and for one of six patients (15.1 \pm 14.6 , median $10 \%$ ) an opioid rotation to keep treatment effects stable have been reported. Reported percentage of patients who develop an opioid-induced hyperalgesia was $10.4 \pm 13.1 \%$ (median 6\%).

For $9.1 \pm 6.0 \%$ (median $8 \%$ ) of CLBP patients, physicians reported the development of signs and clinical correlates suggestive for an opioid use disorder. 


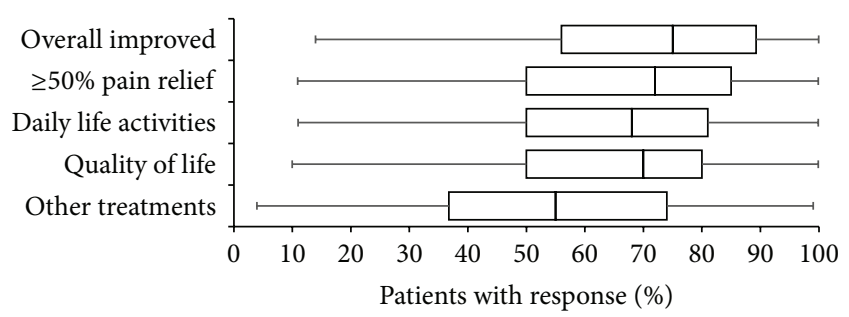

FIGURE 4: Frequency of different effects reported with WHO-step III opioids in patients with chronic low back pain. Parameters shown are box-and-whisker diagrams (with the bottom and top of the box defined by the first and third quartiles, the band inside by the median, and the whiskers representing the 2.5th and 97.5th percentiles).

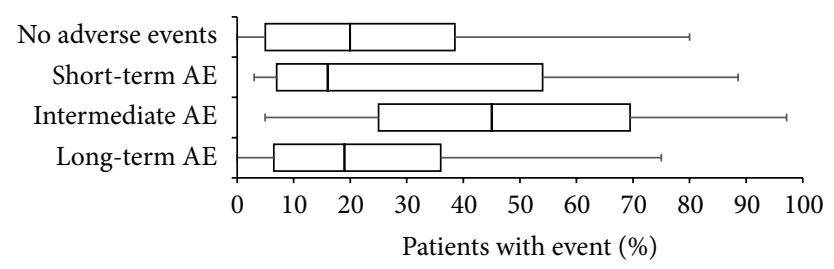

FIGURE 5: Frequency of different adverse effect experiences reported with WHO-step III opioids used for the treatment of patients with chronic low back pain sorted by percentage in descending order. Parameters shown are box-and-whisker diagrams (with the bottom and top of the box defined by the first and third quartiles, the band inside by the median, and the whiskers representing the 2.5th and 97.5th percentiles). Short-term: duration $\leq 2$ weeks; intermediate term: duration 3-8 weeks; long-term: duration $>8$ weeks. AE: adverse event.

\section{Discussion}

Use of WHO-step III opioid analgesics for the management of CNMP and especially CLBP has increased dramatically over the past decade with corresponding increases in negative sequelae and rising concerns about these developments worldwide. Beside pure reaction to the growing population of patients suffering from both conditions, experts frequently criticise an uncritical overprescription of strong opioids by physicians due to an overestimation of beneficial effects, whitewashing of tolerability problems, and denial of critical safety issues.

However, the current analysis of data from a crosssectional German physician survey shows a slightly different picture:

With a median prescription rate of $17 \%$, treatment with WHO-step III opioids is more the exception than the rule for patients suffering from CLBP.

In four of five patients, opioids are combined with other pharmacological treatments and nonpharmacological approaches and in $20 \%$ as part of a multimodal pain management regimen.

Treatment duration is reported to be highly individualized dependent on the extent of beneficial effects and tolerability issues. Nevertheless about one-third

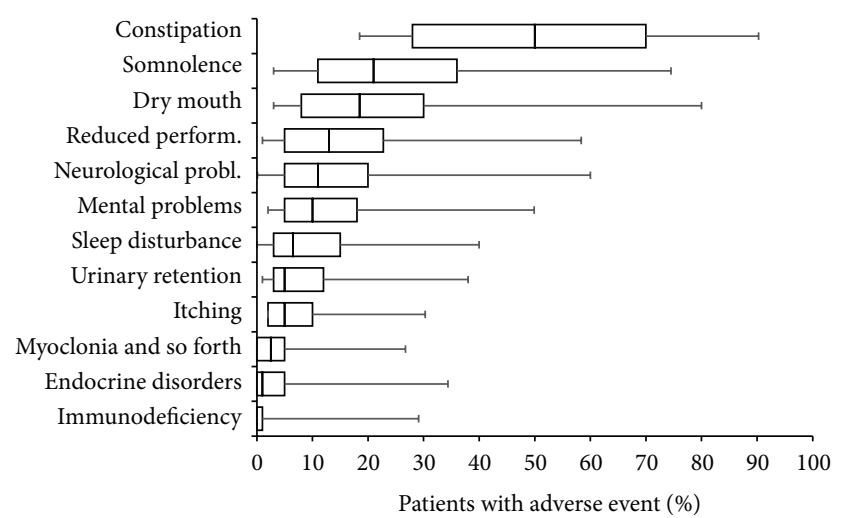

FIGURE 6: Frequency of different side effects reported with WHOstep III opioids used for the treatment of patients with chronic low back pain sorted by percentage in descending order. Parameters shown are box-and-whisker diagrams (with the bottom and top of the box defined by the first and third quartiles, the band inside by the median, and the whiskers representing the 2.5th and 97.5th percentiles).

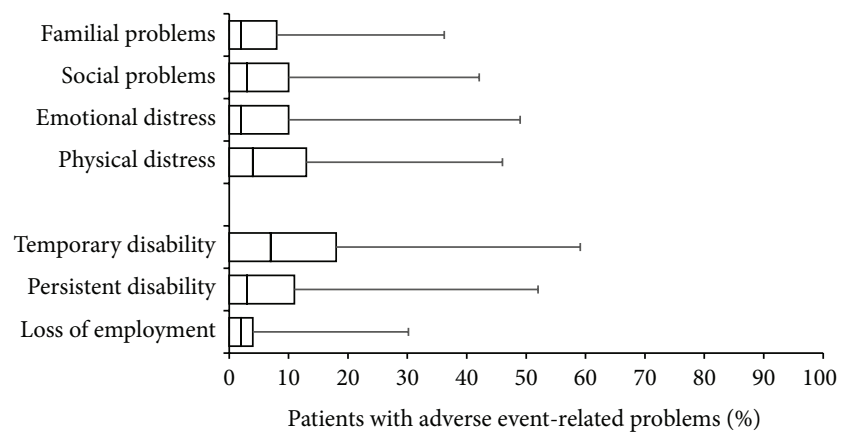

FIGURE 7: Frequency of different side effect-related complaints reported with WHO-step III opioids used for the treatment of patients with chronic low back pain. Parameters shown are box-andwhisker diagrams (with the bottom and top of the box defined by the first and third quartiles, the band inside by the median, and the whiskers representing the 2.5 th and 97.5 th percentiles).

of patients received WHO-step III opioids for more than 12 months.

Overall, treatment effects are substantial, but far below overwhelming. For seven of ten patients, survey participants noted a clinically relevant improvement with respect to their overall situation and for two-thirds of patients they reported significant treatment-related changes, for either pain relief, improvement in daily life activities, or quality of life.

Opioid-related side effects are frequent (only 1 in 5 patients is reported to develop none), are associated with relevant emotional and physical distress ( 1 in 6 ), interfere significantly with familial and social life (1 in 10 ), and lead to transient or persistent disability (1 in 10 and 1 in 16) or even complete loss of employment (1 in 30). 


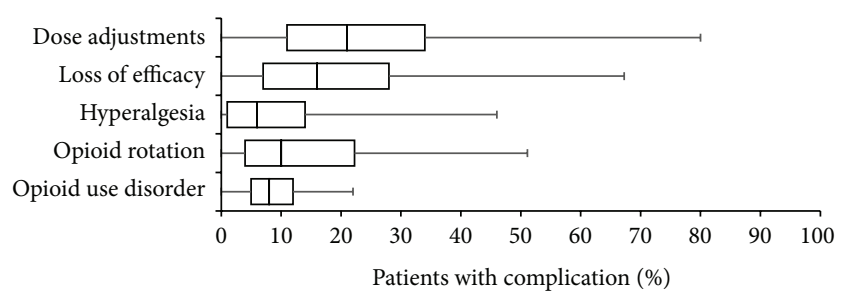

FIGURE 8: Frequency of different application problems reported with WHO-step III opioids for the treatment of patients with chronic low back pain. Parameters shown are box-and-whisker diagrams (with the bottom and top of the box defined by the first and third quartiles, the band inside by the median, and the whiskers representing the 2.5th and 97.5th percentiles).

Proportion of patients with tolerance development and the need for dose adjustments during maintenance treatment (reported for 1 in 4 patients) is rather high, as well as the percentages of patients who present with an untreatable loss of efficacy (1 in 5), those who need an opioid rotation (1 in 6), and those who develop an opioid-induced hypersensitivity ( 1 in $10)$.

With 9 in 100, the proportion of patients with clinical signs suggestive for an opioid use disorder is low, but clinically relevant.

Due to these data, the prescription of WHO-step III opioids to patients with CLBP is obviously not primarily driven by an overestimation of effects and/or a related underestimation of side effects. Reported tolerability and safety data is close to those given in the current literature and particularly the proportion of patients seen at risk for the development of tolerance (treatable or not), opioid-induced hypersensitivity, and OUD by the survey participants have to be taken as a correlate that the prescription of opioids occurs usually being well reflected and with full awareness for critical opioidrelated risk factors by the physicians involved.

Differences between reported effect sizes and effect rates in this survey versus those published for RCTs are predominantly due to the methodological differences of artificial settings under study versus real-life conditions. Compared with data from noninterventional trials with WHO-step III opioids in CLBP, reported pain relief and related effects on daily function as well as quality of life are comparable $[21,22]$. Nevertheless, reported efficacy expectations are high and reflect probably not only objectively verifiable effects, but also related expectations.

As shown by the low percentage of WHO-step III prescriptions, strong acting opioids are more the last than the first choice for patients with CLBP. It can be therefore assumed that physicians prescribe these agents to those CLBP patients only, who showed either none or only insufficient analgesic effects in response to alternative approaches or to those with intolerable side effects of previous pharmacological measures. Under these circumstances, the absolute risk-balance between positive and negative effects of a distinct treatment transforms significantly and becomes relative. If nothing works sufficiently well or well enough to be maintained, or in case prior treatments have to be stopped due to intolerable side effects, physicians are forced to choose alternative measures. In such a situation, strong acting opioids seem, for a number of reasons, as potent alternatives and are worth being considered even for patients with socalled nonmalignant (better noncancer) chronic pain, such as CLBP.

With an average/median of 8.1/7 percent, the proportion of patients with WHO-step III opioids reported in this survey to develop an opioid use disorder is in the middle range of previous reports on this issue. In 1986, Portenoy and Foley reported an estimated addiction risk during chronic opioid treatment in patients with nonmalignant pain of around 5\% on average [23], whereas Fishbain et al. found in a systematic review of 24 articles a prevalence percentage for drug abuse, drug dependence, and drug addiction in the range of 3.2$18.9 \%$ [24]. In addition to a number of methodological differences between both studies and especially to this survey, the currently discussed estimates of addiction risks should be taken with caution, as the reported differences predominantly reflect the lack of a uniquely agreed and valid definition of iatrogenic opioid addiction (i.e., addiction arising during opioid treatment of pain). So far, most of these data reflect a significant spectrum of different definitions and classifications of opioid addiction or opioid use disorder and in most cases the comparability of different risk estimates reported in different studies is rather low, as "iatrogenic opioid addiction" is, what the reporting person says it is, and not what a uniquely agreed consensus statement defines. Nevertheless, the $\sim 9 \%$ prevalence of OUD reported in this survey should be taken as a serious indicator for a significant and probably continuously increasing problem, especially in front of the data of an international working group that reported a nearly threefold increase in OUD-related mortality between 1990 and 2013 [25].

Careful patient selection, variable dose titration, individualized treatment durations, ongoing effect evaluations, knowledge about the broad spectrum of opioid-related side effects, and an increased awareness of physiological as well as psychological complications (such as tolerance development, opioid-induced hypersensitivity, and addiction/opioid use disorder) are essential parameters characterizing a patientcentred use of WHO-step III opioids. Data of this crosssectional physician survey indicate that these key features build (more or less) the fundamentals for a treatment trial with strong opioids. Physicians obviously know about the overall limited efficacy of these drugs and they realize the different tolerability and critical safety risks associated with them. But what kind of alternatives do they have? For more than half of the patients treated with opioids, survey participants reported that "they weren't treatable otherwise." Dramatically increasing numbers of surgical spine interventions and exploding proportions of patients with consecutive failed back surgery syndromes can be taken as additional indicators for the challenging situation; physicians have to manage in case they are confronted with a patient suffering from CLBP in the 21st century. 
Increasing numbers of studies, reports, consensus statements, and guidelines recommend using WHO-step III opioids with knowledge, experience, and caution as well as in a patient-physician relationship built on confidence, partnership, candidness, and time. These factors are critical for the ideal treatment of patients suffering from CLBP. They are essential to tailor different multimodal treatment concepts to the individual needs of affected patients. And they are important to optimize safety, tolerability, and efficacy of potent drugs such as the WHO-step III opioids. If these conditions are clear, guaranteed and agreed between all treatment partners, WHO-step III opioids are a welcome addition to conventional treatment approaches and an important and safe alternative for many patients suffering from CLBP. If not, WHO-step III opioids carry a significant risk to corrupt all other well-intentioned approaches, to worsen the underlying situation, and to harm the patient.

As other studies have shown before and databases currently report, the WHO-step III prescription behaviour of German physicians differs significantly from those of other countries, especially the US. Due to the data of the Pain \& Policy Studies Group of the University of Wisconsin and the WHO Collaborating Center, Germany has worldwide the highest per capita fentanyl consumption rate $(5.7 \mathrm{mg}$; rank \#1) versus only $1.7 \mathrm{mg}$ in the US (rank \#10) [26]. This is also confirmed by our own observation that the transdermal fentanyl patch is the most prevalently prescribed WHO-step III opioid for CNMP in Germany. Reasons for that have been widely discussed and so far no easy answer can be given to explain these discrepancies between the US and Germany. From our point of view, the major reason for the preferential use of fentanyl in Germany is not the opioid compound per se, but the drug-delivery system via a transdermal patch. This combination of an apparently innocent and easy-touse system (the patch) with a potent opioid (fentanyl) has obviously a significant charm for physicians, nursing staff, and patients in Germany.

\section{Strength, Weaknesses, and Limitations}

Obviously, the cross-sectional survey generating the data for this post hoc analysis as well as this analysis suffers from several methodological limitations that should be taken into account when interpreting these results. Typical for such a survey, data were collected retrospectively, reflect personal experience (e.g., internal evidence), and ground predominantly on spontaneous approximations of data about various clinical and pharmacological parameters. Subsequent imprecisions in estimating the percentages for distinct survey parameters are reflected by the high standard deviations observed for the means and the broad spectrum of VAS scores given. Therefore, average values reported in this study must be interpreted with care and only in conjunction with the dispersion data presented in the corresponding box-plots. A prospective observational study design would allow for more accuracy and use of direct measures, of both efficacy (e.g., pain reduction) and tolerability (e.g., assessment of side effects), but would restrict the number of participants (and by that the representativeness of the data) and vice versa increase costs significantly. To get meaningful information from a larger cohort of physicians and to encourage them to participate, the chosen format of a cross-sectional online survey fulfills all prerequisites for an unbiased acquisition of routine data reflecting daily practice.

The large number of participants is one of the major strengths of the survey underlying this post hoc analysis. With 4.283 participants, this physician survey is one of largest ones performed worldwide and despite the considerable data variation, this sample size guarantees a solid basis for any conclusions drawn.

A critical weakness of this survey is the lack of any differential data assessment with respect to the spectrum of currently available and prescribed WHO-step III opioids for CLBP. Therefore all effects, side effects, and safety issues reported and discussed are those for the whole group of strong acting opioids used. Data on preferred prescription rates point out that there might be some safety and efficacy differences between distinct WHO-step III opioids, but with conventional analysis techniques these differences are not evaluable. Nevertheless, the fact that morphine (the so-called gold standard of opioid treatment) is, due to the data of this survey, the least frequently prescribed WHO-step III opioid for patients with CLBP shows that pharmacological advantages and disadvantages of distinct strong acting opioids directly translate into daily use.

\section{Conclusion}

Based on the data from a large cross-sectional survey among 4.283 German physicians, WHO-step III opioids are used as third-line treatment in patients with CLBP, especially in cases where alternative pharmacological and nonpharmacological approaches failed or are associated with significant risks. Treatment characteristics, as well as information given on effect size, side effects, and safety aspects, give insight into a complex multifractal health problem in which opioids represent only one option among several others. Opioid treatment is based on rational thoughts and realistic assumptions with respect to prevalence and size of positive as well as negative effects. Despite a significant percentage of patients with opioid-related side effects, tolerance development, and even signs of OUD, the overall risk-benefit analysis remains positive and in the majority of patients treated with WHOstep III opioids, treatment-related benefits outweigh associated adverse effects.

\section{Conflict of Interests}

The concept for the original CROSSECCO study as well as this post hoc analysis was developed by the Institute for Neurological Sciences (IFNAP) on behalf of the German Pain Association (Deutsche Gesellschaft für Schmerzmedizin, DGS) and the German Pain League (Deutsche Schmerzliga, DSL). The original study was realized by an independent $\mathrm{CRO}$ and sponsored by an unrestricted scientific grant from Mundipharma, Germany. The current post hoc analysis 
was done independently of any financial and/or intellectual influences by the authors. Michael A. Ueberall and Gerhard H. H. Mueller-Schwefe are physicians and independent of any significant/relevant financial or other relationships to the sponsor, except for minor reimbursements for occasional lecture or consulting fees. Alice Eberhardt is an employee of Mundipharma, Germany.

\section{Authors' Contribution}

All authors were involved in drafting the paper or revising it critically for important intellectual content, and all authors read and approved the final paper to be published.

\section{References}

[1] J. B. Braden, M.-Y. Fan, M. J. Edlund, B. C. Martin, A. DeVries, and M. D. Sullivan, "Trends in use of opioids by noncancer pain type 2000-2005 among Arkansas Medicaid and HealthCore enrollees: results from the TROUP Study," Journal of Pain, vol. 9, no. 11, pp. 1026-1035, 2008.

[2] B. D. Sites, M. L. Beach, and M. A. Davis, "Increases in the use of prescription opioid analgesics and the lack of improvement in disability metrics among users," Regional Anesthesia and Pain Medicine, vol. 39, no. 1, pp. 6-12, 2014.

[3] C. S. Zin, L.-C. Chen, and R. D. Knaggs, "Changes in trends and pattern of strong opioid prescribing in primary care," European Journal of Pain, vol. 18, no. 9, pp. 1343-1351, 2014.

[4] I. Schubert, P. Ihle, and R. Sabatowski, "Increase in opiate prescription in Germany between 2000 and 2010: a study based on insurance data," Deutsches Arzteblatt International, vol. 110, no. 4, pp. 45-51, 2013.

[5] H. Jaeschke, "Acetaminophen: dose-dependent drug hepatotoxicity and acute liver failure in patients," Digestive Diseases, vol. 33, no. 4, pp. 464-471, 2015.

[6] T. Stammschulte, W. D. Ludwig, B. Mühlbauer, E. Bronder, and U. Gundert-Remy, "Metamizole (dipyrone)-associated agranulocytosis. An analysis of German spontaneous reports 19902012," European Journal of Clinical Pharmacology, vol. 71, no. 9, pp. 1129-1138, 2015.

[7] A. Lanas, M. A. Perez-Aisa, F. Feu et al., "A nationwide study of mortality associated with hospital admission due to severe gastrointestinal events and those associated with nonsteroidal antiinflammatory drug use," American Journal of Gastroenterology, vol. 100, no. 8, pp. 1685-1693, 2005.

[8] W. B. White, G. Faich, A. Whelton et al., "Comparison of thromboembolic events in patients treated with celecoxib, a cyclooxygenase-2 specific inhibitor, versus ibuprofen or diclofenac," American Journal of Cardiology, vol. 89, no. 4, pp. 425-430, 2002.

[9] European Medicines Agency, "Diclofenac-containing medicines," 2015, http://www.ema.europa.eu/ema/index.jsp?curl=pages/medicines/human/referrals/Diclofenac-containing_medicines/human_referral prac_000009.jsp\&mid=WC0b01ac05805c516f.

[10] European Medicines Agency, "Ibuprofen- and dexibuprofencontaining medicines," July 2015, http://www.ema.europa.eu/ ema/index.jsp?curl=pages/medicines/human/referrals/Ibuprofen_and_dexibuprofen_containing_medicines/human_referral_ prac_000045.jsp\&mid=WC0b01ac05805c516f.

[11] N. Moore, C. Pollack, and P. Butkerait, "Adverse drug reactions and drug-drug interactions with over-the-counter NSAIDs,"
Therapeutics and Clinical Risk Management, vol. 11, pp. 10611075, 2015.

[12] R. Chou, G. J. Fanciullo, P. G. Fine et al., "Clinical guidelines for the use of chronic opioid therapy in chronic noncancer pain," Journal of Pain, vol. 10, no. 2, pp. 113-130, 2009.

[13] R. Chou and L. Huffman, Use of Chronic Opioid Therapy in Chronic Noncancer Pain: Evidence Review, American Pain Society, Glenview, Ill, USA, 2009, http://americanpainsociety.org/uploads/education/guidelines/chronic-opioid-therapy-cncp.pdf.

[14] National Opioids Use Guideline Group (NOUGG), Canadian Guidelines for Safe and Effective Use of Opioids for Chronic Non-Cancer Pain, Version 5.6, 2010, http://nationalpaincentre .mcmaster.ca/documents/opioid_guideline_part_b_v5_6.pdf.

[15] The British Pain Society, Opioids for Persistent Pain: Good Practice. A Consensus Statement Prepared on Behalf of the British Pain Society, the Royal College of Anaesthetists, the Royal College of General Practitioners and the Faculty of Addictions of the Royal College of Psychiatrists, The British Pain Society, London, UK, 2010.

[16] W. Häuser, F. Bock, P. Engeser et al., "Recommendations of the updated LONTS guidelines. Long-term opioid therapy for chronic noncancer pain," Schmerz, vol. 29, no. 1, pp. 109-130, 2015.

[17] N. Sehgal, L. Manchikanti, and H. S. Smith, "Prescription opioid abuse in chronic pain: a review of opioid abuse predictors and strategies to curb opioid abuse," Pain Physician, vol. 15, no. 3, pp. ES67-ES92, 2012.

[18] A. DuPen, D. Shen, and M. Ersek, "Mechanisms of opioidinduced tolerance and hyperalgesia," Pain Management Nursing, vol. 8, no. 3, pp. 113-121, 2007.

[19] M. Lee, S. Silverman, H. Hansen, V. Patel, and L. Manchikanti, "A comprehensive review of opioid-induced hyperalgesia," Pain Physician, vol. 14, no. 2, pp. 145-161, 2011.

[20] G. H. H. Mueller-Schwefe and M. A. Ueberall, "Stellenwert stark wirksamer Opioide aus Behandlersicht: Ergebnisse einer Querschnittbefragung von 4283 opioidverordnenden Ärzten," MMW_Fortschritte der Medizin, vol. 156, no. 5, supplement, pp. 98-105, 2014.

[21] J. H. Lee, S.-C. Yun, J.-G. Lee et al., "The impact of fentanyl matrix on pain and function in spinal disorder-related chronic pain: an open label trial in Korea," Asian Spine Journal, vol. 5, no. 2, pp. 91-99, 2011.

[22] M. A. Ueberall and G. H. Mueller-Schwefe, "Safety and efficacy of oxycodone/naloxone vs. oxycodone vs. morphine for the treatment of chronic low back pain: results of a 12 week prospective, randomized, open-label blinded endpoint streamlined study with prolonged-release preparations," Current Medical Research and Opinion, vol. 31, no. 7, pp. 1413-1429, 2015.

[23] R. K. Portenoy and K. M. Foley, "Chronic use of opioid analgesics in non-malignant pain: report of 38 cases," Pain, vol. 25, no. 2, pp. 171-186, 1986.

[24] D. A. Fishbain, H. L. Rosomoff, and R. S. Rosomoff, "Drug abuse, dependence and addiction in chronic pain patients," Clinical Journal of Pain, vol. 8, no. 2, pp. 77-85, 1992.

[25] GBD 2013 Mortality and Causes of Death Collaborators, "Global, regional, and national age-sex specific all-cause and cause-specific mortality for 240 causes of death, 1990-2013: a systematic analysis for the Global Burden of Disease Study 2013," The Lancet, vol. 385, no. 9963, pp. 117-171, 2014.

[26] Pain \& Policy Studies Group of the University of Wisconsin and the WHO Collaborating Center, 2015, http://www.painpolicy .wisc.edu/global. 


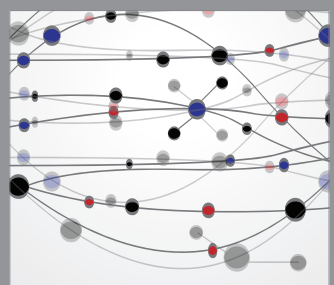

The Scientific World Journal
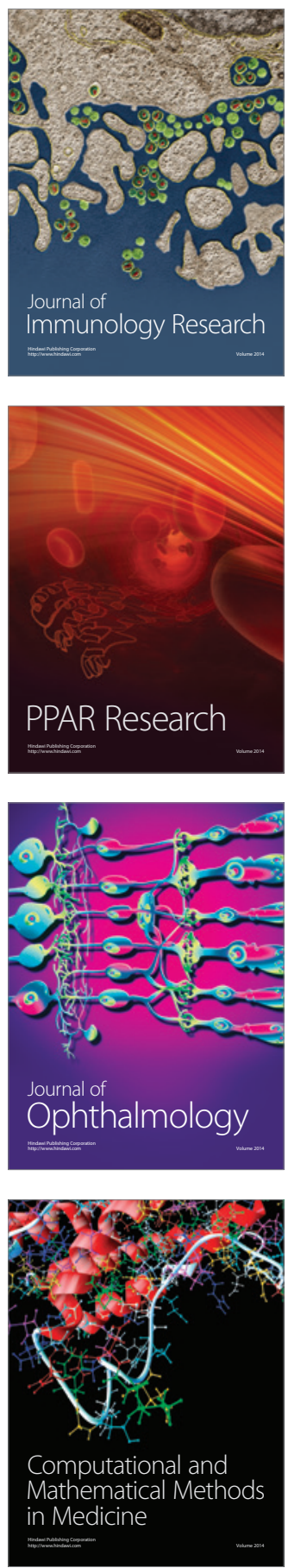

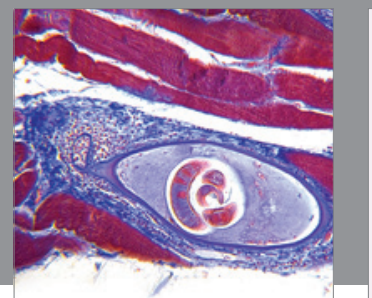

Gastroenterology

Research and Practice
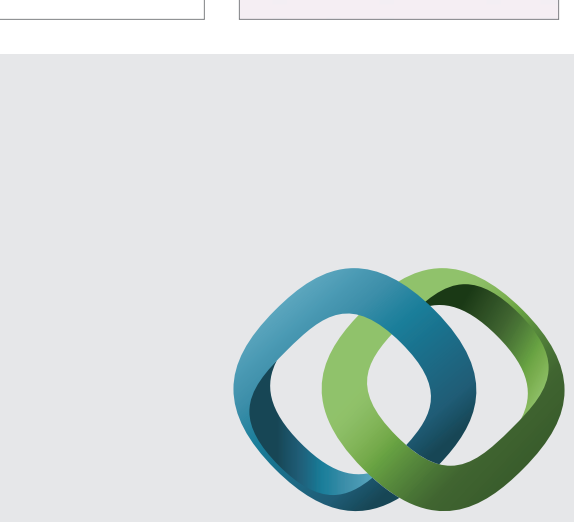

\section{Hindawi}

Submit your manuscripts at

http://www.hindawi.com
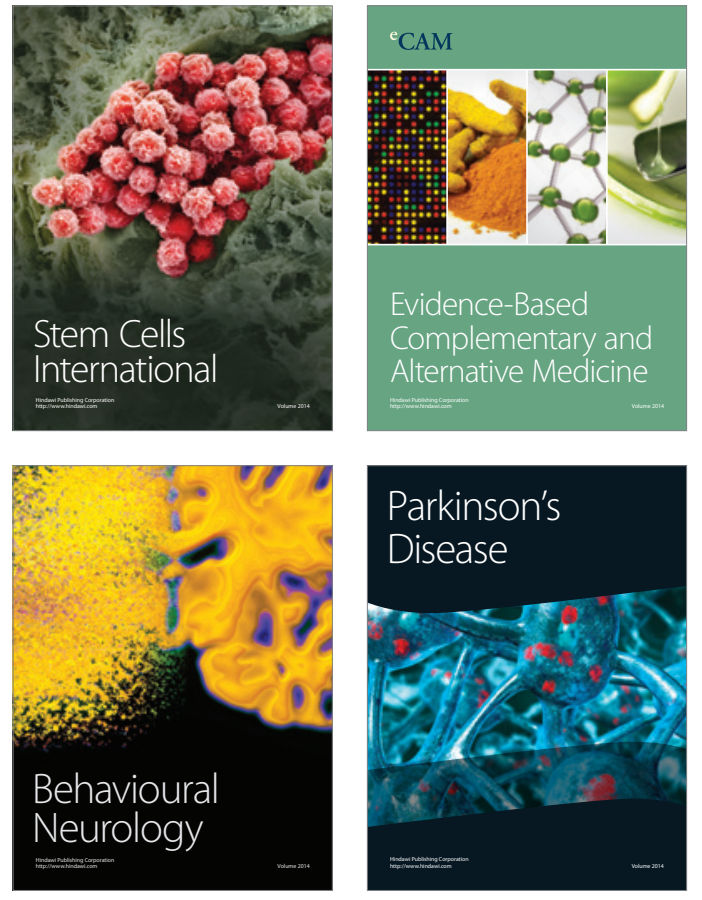
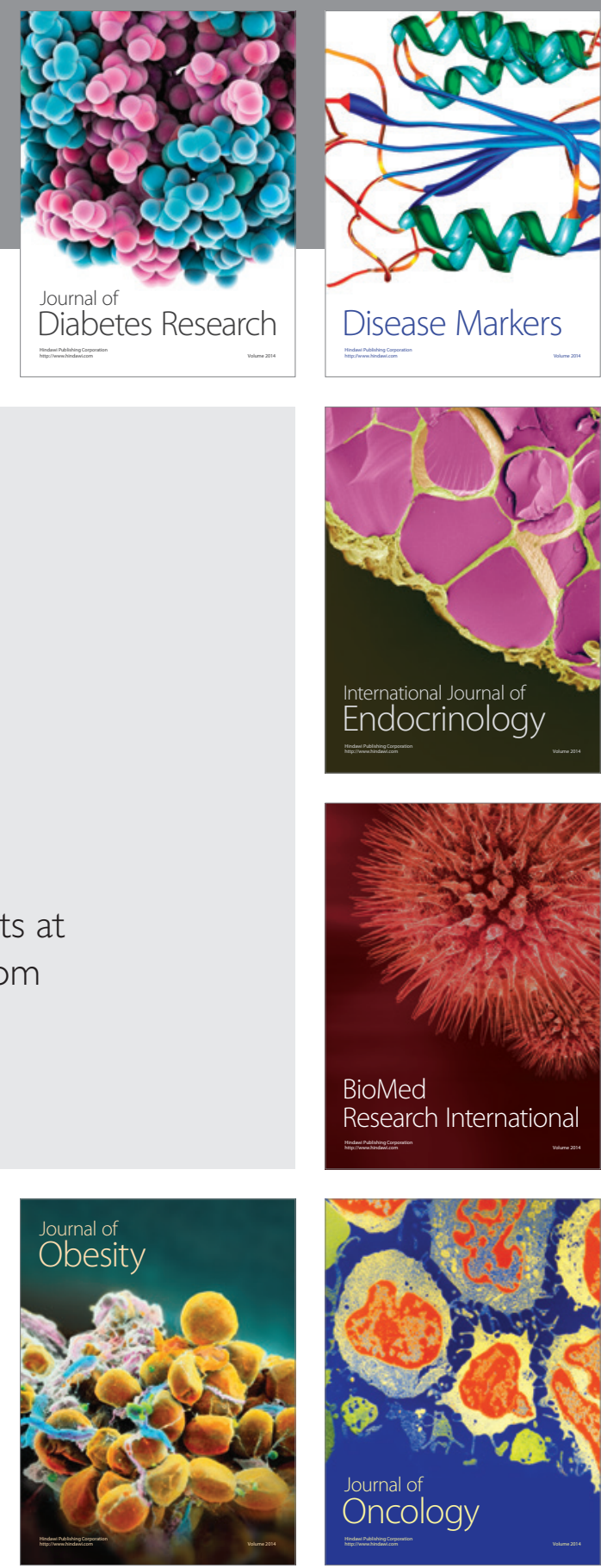

Disease Markers
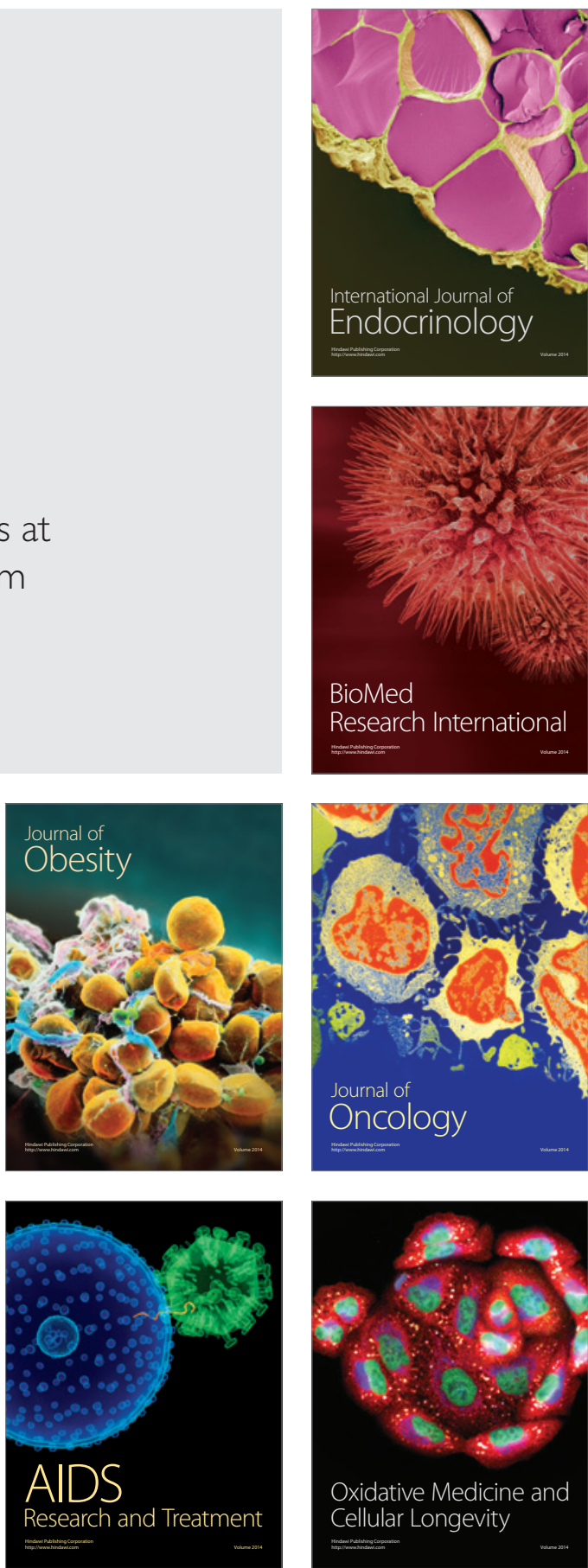